\title{
Femtocell Downlink Power Control based on Radio Environment Maps
}

\author{
Andreas Zalonis, Nikos Dimitriou, Andreas Polydoros \\ Institute of Accelerating Systems \& Applications \\ National Kapodistrian University of Athens \\ Athens, Greece \\ \{azalonis, nikodim, polydoros\}@phys.uoa.gr
}

\author{
Jad Nasreddine, Petri Mähönen \\ Institute for Networked Systems, \\ RWTH Aachen University \\ Aachen, Germany \\ \{jad,pma\}@inets.rwth-aachen.de
}

\begin{abstract}
The paper investigates the use of Radio Environment Maps (REMs) as a tool for Interference Management (IM) in two-tier cellular networks comprising macro- and femto-cells. The REMs are databases that provide, through different instances distributed over network elements, a variety of network- and user-related context information for improving IM and Radio Resource Management (RRM) procedures. In this context, the focus in this paper is to present the benefit of using REM's information on practical power control schemes for the Femtocell DownLink transmission in co-channel two-tier deployment.
\end{abstract}

\section{Keywords-femtocell; power control; Radio Environment Maps}

\section{INTRODUCTION}

The need to increase system capacity in wireless networks has driven the relevant research in the field of wireless communication over the past years. As stated in [1]-[3] a reliable way to increase the capacity is to reduce the distance between the transmitter and the receiver, thus achieving higher quality links. Along these lines, the deployed networks are enhancing their infrastructure with the addition of Femtocells. Femtocells are low power radio access points, providing cellular services to users in the home and small-office environments through the Femtocell Access Points (FAPs). The latter are deployed by the end-users and thus their locations with respect to the Macrocell Base Stations (MBSs) are dynamic and random. Interference Management (IM) and Radio Resource Management (RRM) procedures have the important role to control, with an efficient and robust manner, the various resources (power, channel allocation, rate, etc.) in order for the femtocells to be seamlessly integrated into the network architecture. The optimization target of those procedures varies according to the scenario, which also dictates the assumptions and the constraints.

Channel allocation techniques for femtocells can be classified into two main categories [2]: (i) orthogonal channel allocation and (ii) co-channel allocation. In the first category, different sets of sub-channels are given to femtocells and macrocells for RRM. These sets can be fixed (e.g. depending on the geographical area) or dynamic (e.g. depending on user mobility or traffic load). In the second category the same pool of sub-channels is used for both macrocells and femtocells, thus generating interference between the two layers. Although it is more spectrum and power efficient, co-channel allocation in two-tier networks is a very challenging task, which dictates the need for advanced IM approaches ([2]-[7])

IM techniques traditionally use collected data from users (feedback) and static network planning information. However, because of the progress in hardware and software, next generation terminals and access points are expected to be able to collect and store more diverse and rich information compared to the existing equipments. The stored information can be used by IM and RRM techniques to enhance their effectiveness. A Radio Environment Map (REM) [8] has been envisaged as an integrated information structure that involves various types of information and data models such as geolocation data, path loss and shadowing models, interference maps, available services, spectral usage regulations, locations and activities of radio nodes, user and service policies, assessment of past decisions, etc. This amount of diverse context information offers opportunities for a more efficient allocation of the available radio resources among the users and more effective interference control. The challenge resides in the exploitation of the REM context information (e.g. PHY measurements, power profiles, topological data, directional spectrum sensing data, measured interference etc.) for the development of pragmatic IM or RRM algorithms by keeping the complexity as simple as possible. Different instances of the REMs with different types of information can be stored at various levels of the network infrastructure [9] (in the FAP, the MBS, or even a dedicated controller).

In this paper, the use of "rich" context information stored in REMs is considered in order to enhance the performance of pragmatic Power Control (PC) algorithms for the FAP's downlink transmission in co-channel femtocell deployments. In particular, we consider a 3GPP Long Term Evolution (LTE) network [10] with femtocells. The scenario under investigation is presented in section II. Different levels of stored information are considered and discussed in section III, where the downlink PC strategies are described. In section IV the results of the assessment are presented and discussed. The objective is to highlight and assess the benefits of using REMs in controlling the interference in two-tier networks.

This work has been supported by the FARAMIR (ICT-248351) and ACROPOLIS (ICT-257626) projects funded by the European Commission. 


\section{SCENARIO}

In [1] the Femto Forum identified the various scenarios where interference impacts the performance of a two-tier network when co-channel allocation is considered. Herein, we will focus on the downlink interference from the FAP to the Macrocell User Equipment (MUE). In this scenario the MUE is located in the same area with the FAP and uses the same channel for the downlink communication with the MBS. Closed access FAP is considered, which means that the MUE cannot use the FAP to access the network. The MUE can be inside the house where the FAP is deployed, or it can be outside in a small distance from it. The interference from the FAP will result in coverage holes in the macrocell where the MUE will not have the necessary SINR to communicate with the MBS. In Figure 1. the selected interference scenario is depicted.

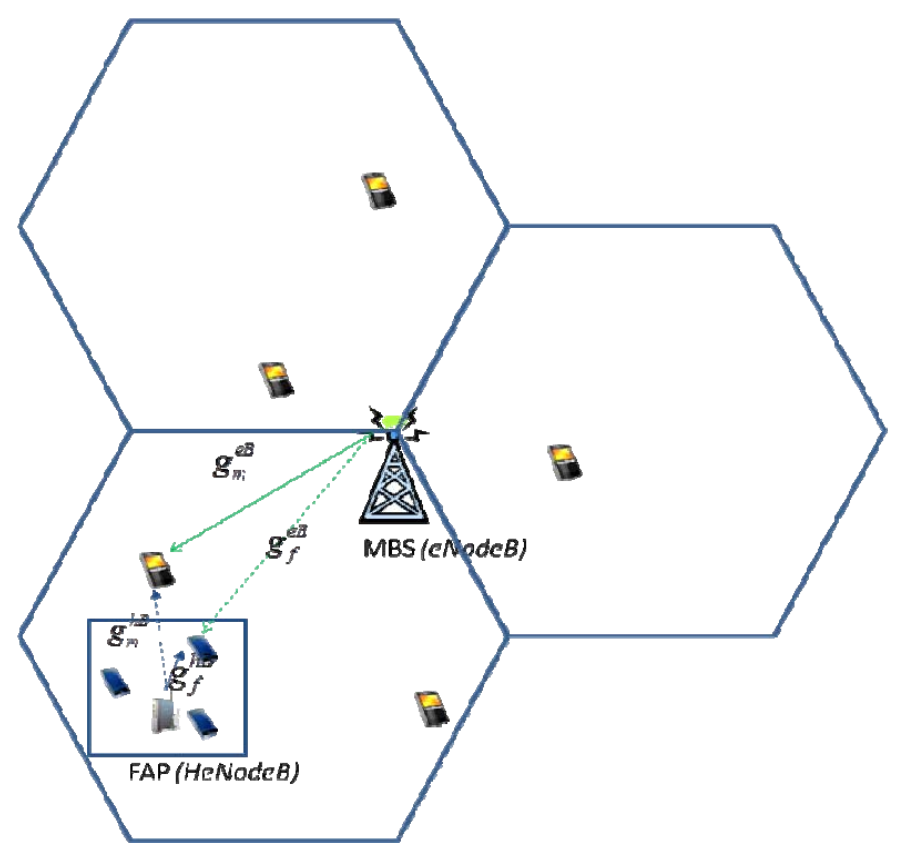

Figure 1. Femtocel to Macrocell UE interference

The macrocell is considered as a primary infrastructure, and the femtocell as a secondary one. The focus in this study is to assess the effectiveness of the protection that can be provided to the MUE from the FAP's downlink interference with the use of downlink PC, without considering the effect to the FAP connections to the Femtocell User Equipments (FUEs).

\section{REM-BASED POWER CONTROL}

The main difference between the REM-based PC approach and the traditional ones is that the information used by the optimization algorithm is not only related to Channel State Information (CSI) feedback from a UE to its serving BS, but also includes information obtained by accessing databases at various levels of the infrastructure [9], where the information is collected using different approaches (sensor networks, localization techniques [11], [12], etc).

In this scenario various levels of context information can be exploited from a REM-based PC procedure. A first level of information is the geolocation knowledge, without any provision for the existence or not of a neighborhood victim MUE. For example the relevant location of the FAP and the MBS can be used for distance-based power control, where the FAP transmits at selected power levels based on its distance to the MBS (higher level close to the MBS and lower level close to the cell edge [15]). When no MUE is present this method results in unnecessary loss of the femtocell throughput and at the same time it does not guarantee a level of protection for a victim MUE.

A second level of information is the knowledge of the location of both the FAP and the MBS, plus the knowledge of the existence of a neighborhood victim MUE (without the information of its exact location). In this case the FAP selects the appropriate transmission power in order to reduce the interference to MUE based on some pre-defined assumptions. This approach was followed in [13] and [14] for $3 \mathrm{G}$ and in [15] for LTE femtocells, where the following algorithm was proposed for the protection of the MUE (we will refer to this as the baseline in the rest of the paper):

- The FAP (HeNB) measures the Reference Signal Received Power (RSRP) and the SINR from the macro BS and neighboring FAPs.

- Upon detection of a neighboring victim MUE the FAP reduces its transmission power aiming to maintain a predefined SINR target for the MUE (calculated by using a fixed predefined assumption for pathloss distance)

- In case the target cannot be achieved, the FAP transmits at a predefined minimum power level.

In this algorithm, various assumptions had to be taken into consideration. Because of the lack of direct communication between the victim MUE and the FAP, the exact downlink received power measured at the MUE from its connected MBS is unknown to the FAP. In [15] it was proposed that the FAP will measure its received power (RSRP) from the MBS, and because of the FAP's small coverage area, a close by MUE is assumed to have similar RSRP measurements. Another assumption was a fixed path loss distance in $\mathrm{dB}$ between the MUE and the FAP for the calculation of the interference ( 80 $\mathrm{dB})$. This algorithm has been shown in [15] to reduce MUE outage with respect to the case when the existence of a neighborhood victim MUE is unknown.

A third level of information is the location of both the FAP and the MBS, and also the knowledge of the existence and the location of a neighborhood victim MUE. In order to exploit the advantage of knowing the location of the MUE, the information for the path loss and shadowing models of the area should also be stored in the REM allowing the FAP to estimate the expected MUE's SINR. The REM-based procedure can then be described in the following way:

- The FAP accesses the REM to update the available path loss and shadowing models for its area (based on building characteristics and geographical data) and the 
positions of the other neighboring network elements (MBS, FAPs, MUEs).

- After detecting the presence of a neighboring victim MUE, the FAP calculates its transmission power aiming to maintain a predefined SINR target for the MUE by using the context information collected in the first step.

- In case the SINR target cannot be met, FAP transmits at a predefined minimum power.

In Figure 1. the 2-tier network setup is presented with one macro- and one femto-cell. The calculation of the interference is based on the following parameters:

$m=1,2, \ldots, M$ macrocell users

$f=1,2, \ldots, F$ femtocell users

$g_{x}^{y}$ : Total channel gain between $\mathrm{x}$ and $\mathrm{y}$,

where $\mathrm{x}: f, m$ and $\mathrm{y}: e B$ (MBS), $h B$ (FAP)

The total channel gain $g_{x}^{y}$ can be decomposed into a path loss (distance-depended), a shadowing, and a fast fading term:

$g_{x}^{y}=L\left(a,\left|x_{y}-x_{x}\right|\right) S\left(x_{y}, x_{x}\right) h_{x}^{y}$

where

$P L(d)$ : Pathloss for distance $\mathrm{d}$,

example $\mathrm{d}=\left|x_{e B}-x_{m}\right|$, path loss exponent: $\alpha$

$S(d)$ : Shadowing term

$h_{x}^{y}:$ fast fading term

In the proposed REM-based PC algorithm we have the following target for the MUE user m:

$$
\frac{p_{m}^{e B}\left|g_{m}^{e B}\right|^{2}}{\hat{p}^{h B}\left|g_{m}^{h B}\right|^{2}+\sigma^{2}}=\operatorname{SINR}_{m}^{e B} \geq \gamma_{m}^{e B}
$$

We assume a fixed transmission power $p_{m}^{e B}$ from the MBS and an accurate knowledge of the path loss and the shadowing components. Based on this information we can calculate from

(1) the FAP's transmit power $\hat{p}^{h B}$.

The accuracy and the level of the available context information depend on the REM's implementation and deployment scenario. For example, it is very difficult to store accurate fast fading information for the UEs, since as shown in [16], in a typical OFDMA cellular network the BS - based on the CSI feedback - receives outdated information for the real channel of an UE of about 3-4 frames.

In the above REM-based PC algorithm we assumed complete knowledge of the network components' locations, the path loss and shadowing terms. While the location of MBS is known in advance, the FAP location needs to be estimated since the user may place the FAP randomly inside the house. The victim MUE's position relative to the FAP and the building also has to be estimated. We assume herein that these locations are determined either from the FAP and MUEs themselves, or from a dedicated sensor network deployed near the house from the operator. The path loss term for the calculation of the channel gains between the network elements is also assumed to be stored in the REM for a specific area based on long term operator's measurements. A sensor network could also be used to estimate the shadowing term, but this is not an easy task and it may result in inaccurate estimates. Assuming that the victim MUE's distance to the FAP is smaller than the MBS shadowing correlation distance, the FAP will be able to estimate the MBS-to-MUE shadowing term based on its power measurements from the MBS. For the FAP-to-MUE shadowing term - on the other hand - we have to rely on the accuracy of the sensing results.

Here we propose a modified version of the REM-based PC where the FAP-to-MUE shadowing term is unknown and we only know the statistics of the shadowing, which is a lognormal distribution with zero mean and known standard deviation $\sigma$ that characterizes the area. In this case we use this knowledge to calculate a back-off term for the total channel gain estimate between the FAP and the MUE based on a target outage level [17]:

$$
B O=\sqrt{2} \sigma \operatorname{erfinv}\left(1-2 F_{o}\right)
$$

In the rest of the paper we assume that the standard deviation for the femtocell area is $4 \mathrm{~dB}$ (Stored in REM). Thus for an outage of $2 \%$ in the channel gain estimate based on (2) we have a Back-Off (BO) term of about $-8 \mathrm{~dB}$. In the rest of the paper we will refer to this version of the algorithm as REM-based-PL (Path-Loss term only).

\section{ASSESSMENT}

The objective here is to highlight the potential gain of using the rich context information in a two-tier network deployment. This gain can serve as an upper bound for pragmatic REMbased algorithms, considering that the inaccuracies in the stored REM's data will result in reduced performance figures. The metric used for assessment is the MUE outage that is observed when the FAP transmits at a power level such that due to the generated co-channel interference - the MUE cannot satisfy its SINR target.

Specifically we compare the baseline PC based on the detection of the presence of a victim MUE [15], with the REM-based PC, with the location, the path loss and the shadowing knowledge. We also examine the more realistic REM-based-PL where no shadowing information is available in the REM (only the standard deviation). TABLE I. summarizes these approaches.

TABLE I. POWER CONTROL StRATEGIES

\begin{tabular}{|l|l|l|l|}
\hline \multirow{2}{*}{} & \multicolumn{3}{|c|}{ Power Control } \\
\cline { 2 - 4 } & \multicolumn{1}{|c|}{$\begin{array}{c}\text { 1. Baseline } \\
\text { ([15]) }\end{array}$} & \multicolumn{1}{|c|}{$\begin{array}{c}\text { 2. REM-based- } \\
\text { PL }\end{array}$} & \multicolumn{1}{|c|}{ REM-based } \\
\hline $\begin{array}{l}\text { Context } \\
\text { information } \\
\text { used }\end{array}$ & $\begin{array}{l}\text { MBS, FAP } \\
\text { location, MUE } \\
\text { detection }\end{array}$ & $\begin{array}{l}\text { MBS, FAP } \\
\text { location, MUE } \\
\text { detection and } \\
\text { location, path } \\
\text { loss term }\end{array}$ & $\begin{array}{l}\text { MBS, FAP } \\
\text { location, MUE } \\
\text { detection and } \\
\text { location, path } \\
\text { loss term, } \\
\text { shadowing term }\end{array}$ \\
\hline
\end{tabular}


The simulation setup is based on the OFDMA Interference Scenario Evaluation Methodology for LTE femtocells presented in [15] for the suburban case. The parameters used in our simulation are summarized in TABLE II.

TABLE II. SIMULATION PARAMETERS

\begin{tabular}{|l|l|}
\hline \multicolumn{1}{|c|}{ Parameter } & \multicolumn{1}{|c|}{ Assumption } \\
\hline Cell Layout & Hexagonal grid, 3 sectors per cell \\
\hline Inter-MBS distance & $1732 \mathrm{~m}$ \\
\hline Carrier Frequency & $2000 \mathrm{MHz}$ \\
\hline LTE bandwidth & $10 \mathrm{MHz}$ \\
\hline $\begin{array}{l}\text { MBS Shadowing standard } \\
\text { deviation }\end{array}$ & $8 \mathrm{~dB}$ \\
\hline $\begin{array}{l}\text { MBS Auto-correlation } \\
\text { distance of shadowing }\end{array}$ & $50 \mathrm{~m}$ \\
\hline Wall penetration loss & $10 \mathrm{~dB}$ \\
\hline MBS Tx power & $46 \mathrm{dBm}$ \\
\hline MUE Noise Figure & $9 \mathrm{~dB}$ \\
\hline $\begin{array}{l}\text { Minimum distance between } \\
\text { MUE and BS }\end{array}$ & $35 \mathrm{~m}$ \\
\hline MUE distribution & $\begin{array}{l}\text { Uniformly distributed within a circle } \\
50 \mathrm{~m} \text { around the FAP }\end{array}$ \\
\hline $\begin{array}{l}\text { Minimum distance between } \\
\text { MUE and FAP }\end{array}$ & $20 \mathrm{~cm}$ \\
\hline Min/Max Tx FAP Power & $-10 / 20 \mathrm{dBm}$ \\
\hline $\begin{array}{l}\text { FAP Auto-correlation } \\
\text { distance of shadowing }\end{array}$ & $3 \mathrm{~m}$ \\
\hline Thermal noise density & $-174 \mathrm{dBm} / \mathrm{Hz}$ \\
\hline Simulation Scenario & Suburban \\
\hline House size & $12 \times 12 \mathrm{~m}$ \\
\hline Number of victim MUEs & 1 \\
\hline Antenna pattern & Seent FAP inside the \\
\hline
\end{tabular}

\section{A. Illustrative examples}

In this subsection two characteristic examples of femtocell deployments are presented. In the first case the femtocell is deployed in an area where the macrocell signal is weak, while in the second case the femtocell is deployed in an area with a strong macrocell signal. The baseline and the REM-based strategies are compared (strategies 1 and 3 from TABLE I. . The SINR target in this example is set to $\gamma_{m}^{e B}=3 \mathrm{~dB}$. The objective here is to identify the conditions where the use of the REM information is more beneficial for the protection of the MUEs.

Femtocell in an area with relatively weak macrocell signal: This is the case according to which the femtocell is deployed in an area at the edge of the cell and/or outside of the center direction of the antenna beam. We simulated 1000 MUE random positions in the vicinity of the FAP (inside a circle around the FAP of radius $50 \mathrm{~m}$ ) and calculated the average outage experienced from the MUE. For the REMbased PC the measured outage was calculated at $35.1 \%$, while for the baseline PC it was $45.5 \%$, therefore we observed a gain of about $10 \%$.

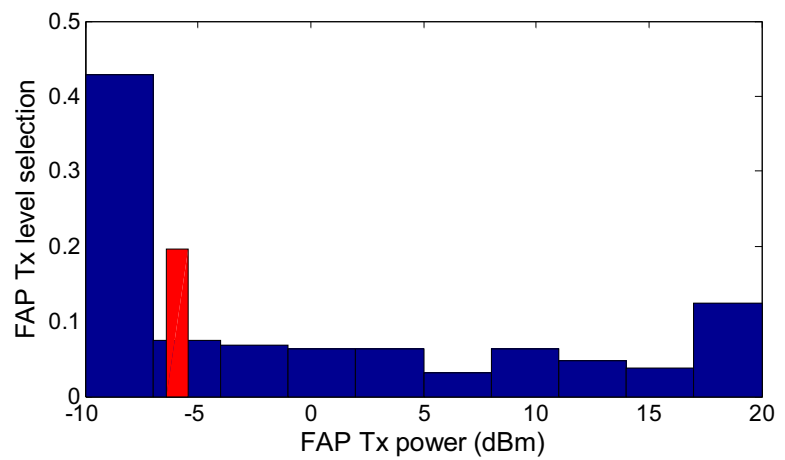

Figure 2. FAP Tx Power profile in weak macro-signal

The FAP Tx power distribution based on the REM-based $\mathrm{PC}$ (in blue) is depicted in Figure 2. In the same figure the red column depicts the constant $\mathrm{Tx}$ power of $-6 \mathrm{~dB}$ calculated from the baseline $\mathrm{PC}$ based on the predefined assumptions for the channel gain estimates. We see that the REM-based PC algorithm was forced to select the minimum Tx level more than the $40 \%$ of the times causing the increased outage. This highlights the fact that any minimal provision for the FAP coverage, which is represented in our scenario with the minimum Tx power of $-10 \mathrm{dBm}$, will inevitably result in high probability of outage for the victim MUE. That means that if we want to have an acceptable outage for the MUE in this area we have to reduce the Tx power level below the minimum Tx level of $-10 \mathrm{~dB}$.

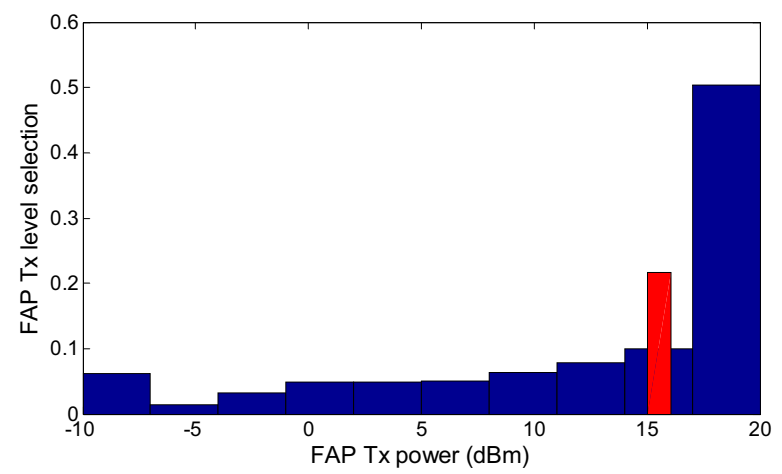

Figure 3. FAP Tx Power profile in strong macro-signal

Femtocell in an area with relatively strong macrocell signal: This is the case when the femtocell is deployed in an area at the center of the sector's antenna beam and/or relatively close to the MBS. The measured outage now was found to be equal to $4.5 \%$ for the REM-based PC and $45.8 \%$ for the baseline PC. The reason for this increased gain of REM-based PC over the baseline is the significantly lower selection of the minimum Tx level (less than 10\%; see Figure 3. ), since the victim MUE receives strong signal from the MBS and therefore the FAP power margin is larger. 


\section{B. Overall comparison}

In Figure 4. we present the average MUE outage when we have random femtocell deployment in rings around the MBS, for the three PC algorithms presented in section III, and for three different SINR targets for the MUE (-6, 3, $9 \mathrm{~dB})$. Here the aim is to average out the effects of MBS shadowing and the antenna pattern; thus the distance from the MBS will be the only parameter that will characterize the MBS signal strength.

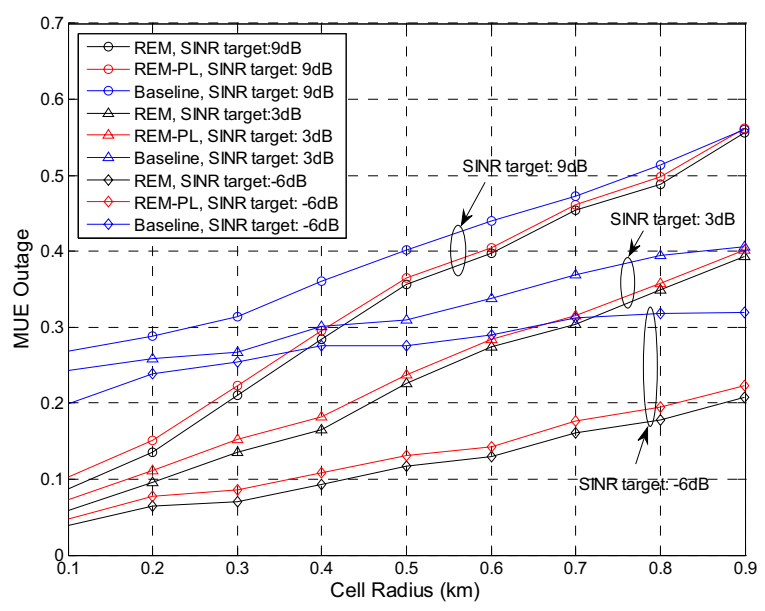

Figure 4. MUE Outage vs. distance

The benefit of using "rich" context information is clearly presented in this figure. As the femtocell is deployed closer to the cell edge, the outage gain is reduced. This is expected since the lower threshold for the FAP Tx power has a bigger effect when the MBS signal is weaker. This is most evident in the most demanding SINR target of $9 \mathrm{~dB}$. Also as expected, the outage increases as the MUE's SINR target increases, since the interference generated by the FAP has a bigger effect in the SINR calculation. The REM-based-PL algorithm achieves a clear gain in the MUE's measured outage over the baseline algorithm, and it is slightly worst from the ideal REM-based algorithm. The difference in the outage from the baseline is mainly attributed to the knowledge of the location, since - for example - the path loss term varies substantially if the MUE is inside or outside the house. The small difference in the outage of the REM-based-PL versus the ideal REM-based is accomplished because of the conservative approach of using a fixed back-off loss in the estimation of the channel gain between the FAP and the MUE. This approach has the drawback that the FAP is forced to transmit at a lower level than needed to protect the MUEs which results in femtocell coverage loss.

\section{CONCLUSIONS}

The potential gain in resource allocation by using the REM context information was presented. An ideal REM-based PC was proposed and compared with a baseline PC algorithm designed for LTE femtocell deployment scenarios. It was shown that the use of stored context information increases the effectiveness of a PC algorithm to protect a co-channel MUE. A version of the REM-based PC without the knowledge of the shadowing term was also proposed and compared to the previous algorithms. The estimation of the shadowing term with the use of a back-off term, based on the standard deviation, was shown to protect the MUE almost as good as the ideal case, at the expense of a lower FAP's transmission power level that may lead to smaller femtocell coverage.

\section{REFERENCES}

[1] www.femtofotum.org: "Interference Management in UMTS Femtocells", December 2008.

[2] D. Lopez-Perez, et al., "OFDMA Femtocells: A Roadmap on Interference Avoidance”, IEEE Communication Magazine, Sept. 2009.

[3] V. Chandrasekhar, J. G. Andrews, "Femtocell Networks: A Survey", IEEE Communication Magazine, Sept. 2008

[4] Vikram Chandrasekhar, Jeffrey G. Andrews, Tarik Muharemovic, Zukang Shen, Alan Gatherer,"Power Control in Two-Tier Femtocell Networks", IEEE Transactions on Wireless Communications, vol. 8, no. 8, August 2009

[5] Ismail Guvenc, Moo-Ryong Jeong, Fujio Watanabe, and Hiroshi InamuraA, "Hybrid Frequency Assignment for Femtocells and Coverage Area Analysis for Co-Channel Operation", IEEE Communications Letters, vol. 12, no. 12, December 2008

[6] Holger Claussen, "Performance of Macro- And Co-Channel Femtocells In A Hierarchical Cell Structure", IEEE PIMRC 2007, Athens, Greece, Sept. 2007.

[7] Talha Zahir, Kamran Arshad, Youngwook Ko and Klaus Moessner, "A Downlink Power Control Scheme for Interference Avoidance in Femtocells", IWCMC 2011, Istanbul, Turkey, 2011.

[8] Y.Zhao, B.Le, J.H.Reed, "Network Support: The Radio Environment Map", Chapter 11 in Cognitive Radio Technology - 2nd Edition (ed. B.Fette), Butterworth-Heinemann Publ., March 2009.

[9] T. Cai, J. van de Beek, B. Sayrac, S. Grimoud, J. Nasreddine, J. Riihijärvi and P. Mähönen, "Design of Layered Radio Environment Maps for RAN Optimization in Heterogeneous LTE Systems," IEEE PIMRC 2011, Toronto, Canada, September 2011.

[10] 3GPP TS 36.300 v8.0.0, "Evolved Universal Terrestrial Radio Access (E-UTRA) and Evolved Universal Terrestrial Radio Access Network (EUTRAN); Overall description; Stage 2".

[11] N.Miliou, A. Moustakas, A.Polydoros, "Interference Source Localization and Transmit Power Estimation under Log-Normal Shadowing", Proceedings of EUSIPCO 2011, Barcelona, Spain, September 2011.

[12] J. Nasreddine, N. Miliou, J. Riihijärvi, A. Polydoros, P. Mähönen, "Using Geolocation Information for Dynamic Spectrum Access in Cellular Networks," $6^{\text {th }}$ ACM Workshop on Performance Monitoring and Measurement of Heterogeneous Wireless and Wired Networks ( $\mathrm{PM}^{2} \mathrm{HW}^{2} \mathrm{~N}$ 2011) in conjunction with MSWIM 2011.

[13] Mehmet Yavuz, Farhad Meshkati, Sanjiv Nanda, Akhilesh Pokhariyal, Nick Johnson, Balaji Raghothaman and Andy Richardson, "Interference Management and Performance Analysis of UMTS/HSPA+ Femtocells", IEEE Communications Magazine, September 2009.

[14] 3GPP TR 25.967 8.0.1 (2009-03), Home Node B Radio Frequency (RF) Requirements (FDD) (Release 8), 2009.

[15] www.femtofotum.org: "Interference Management in OFDMA Femtocells", March 2010.

[16] IEEE 802.16 Broadband Wireless Access Working Group, Task Group m: "IEEE 802.16m Evaluation Methodology Document (EMD)"

[17] J. Nasreddine, J. Riihijarvi, and P. Mahonen, "Location-based adaptive detection threshold for dynamic spectrum access," in the 4th IEEE International Symposium on New Frontiers in Dynamic Spectrum Access Networks (DySPAN 2010), Singapore, April 2010. 